\title{
Single-molecule Detection of Fluorescence Resonance Energy Transfer Using Confocal Microscopy
}

\author{
Sung Hyun Kim, Don Seong Choi, and Doseok Kim* \\ Department of Physics and Interdisciplinary Program of Integrated Biotechnology, Sogang \\ University, Seoul 121-742, Republic of Korea
}

(Received February 29, 2008 : revised May 21, 2008 : accepted May 23, 2008)

\begin{abstract}
We demonstrated single-molecule fluorescence resonance energy transfer (FRET) from single donor-acceptor dye pair attached to a DNA with a setup based on a confocal microscope. Singlestrand DNAs were immobilized on a glass surface with suitable inter-dye distance. Energy transfer efficiency between the donor and the acceptor dyes attached to the DNA was measured with different lengths of DNA. Photobleaching of single dye molecule was observed and used as a sign of single-molecule detection. We could achieve high enough signal-to-noise ratio to detect the fluorescence from a single-molecule, which allows real-time observation of the distance change between single dye pairs in nanometer scale.
\end{abstract}

Keyword: single-molecule detection, fluorescence, FRET, confocal microscopy

OCIS codes : (300.0300) Spectroscopy; (180.0180) Microscopy; (170.6280) Spectroscopy, fluorescence and luminescence.

\section{INTRODUCTION}

Fluorescence Resonance Energy Transfer (FRET) is an energy transfer process between fluorescent dye molecules [1]. Due to its high sensitivity to the change of distance between the dyes, it has received great attention for the study of biological systems [2]. FRET technique has a spatial resolution of sub-nanometer, and can be used as a probe of inter- and intra-molecular dynamics of biological macromolecules such as protein conformational change, enzyme-substrate reaction, and RNA folding [2-4]. Determination of FRET efficiency in ensemble measurement is, however, not straightforward due to the difficulty in distinguishing FRET pairs from unpaired dyes or to the dyes not properly labeled to the biomolecule in question that might give huge background [5]. By contrast, single-molecule detection provides a method that is free from this obstacle as it can only collect the signal from one single FRET pair. Another advantage of this technique is that it allows monitoring the structural- or conformation change of single molecules in situ [6]. Here we demonstrated single-molecule detection of FRET from a dye-pair attached to a single- stranded DNA using confocal microscopy.

\section{THEORETICAL BACKGROUND}

Forster Resonance Energy Transfer (FRET), named after the German physicist 'Theodor Forster', is a phenomenon of the energy transfer between two chromophores via dipole-dipole interaction [1]. As the chromophores involved are usually fluorescent dye molecules, it is commonly called Fluorescence Resonance Energy Transfer. The FRET efficiency, $E$, defined as the quantum yield of the energy transfer transition, has the following dependence on the distance $r$ between the two fluorescent dye molecules, referred to as a donor and an acceptor.

$$
E=\frac{1}{1+\left(r / R_{0}\right)^{6}}
$$

Here, $\mathrm{R}_{0}$ is a Forster radius, defined as the distance between two fluorescent molecules for which the FRET efficiency is $50 \%$. In practice, apparent FRET efficiency given by [7]

\footnotetext{
*Corresponding author: doseok@sogang.ac.kr
} 


$$
E=\frac{F_{A}}{F_{D}+\gamma F_{A}}
$$

is used to calculate FRET efficiency from the measured fluorescence intensities as it is a good approximation for true FRET efficiency, where $\gamma$ is a parameter representing relative detection efficiencies and quantum yields of the two dyes, and $\mathrm{F}_{\mathrm{A}}$ and $\mathrm{F}_{\mathrm{D}}$ are the emission intensity of the donor and acceptor, respectively. For the Cy3 (donor) and Cy5 (acceptor) dyes, which were used throughout this study, the correction factor $\gamma$ was approximately 1 in our experimental setup.

\section{MATERIAL AND METHODS}

\section{Confocal microscopy}

A confocal microscope built around a commercial inverted microscope (Olympus, IX51) was used to achieve high S/B ratio for the single-molecule detection (Figure 1(a)) [8]. $532 \mathrm{~nm}$ laser (CrystaLaser) beam was sent through an objective lens (Olympus, 1.2 NA, water immersion) and focused on the surface of a cover slip on which the partial duplex DNA was attached. Fluorescence signals from the donor and acceptor were collected by the same objective lens and separated spectrally by a dichroic mirror (Omega). Before the fluorescence lights were focused at the ends of corresponding optical fibers to be guided to avalanche photodiodes (APD, Perkin Elmer, SPCM-AQ4C), emission filters for $\mathrm{Cy} 3$ and $\mathrm{Cy} 5$ (Omega) were inserted to block the scattered laser light. The fluorescence was focused by a lens $(\mathrm{f}=100 \mathrm{~mm}$ ) on a tip of a multi-mode optical fiber to deliver the photons to the APD. As the core size was $62.5 \mu \mathrm{m}$, it is used as a confocal pinhole. Each single photon measured by the APDs was converted to electrical pulses and counted by a counting board (National Instruments, 6602). To find DNA molecules attached on the surface of the cover slip, we scanned the sample with piezo-electric translation stage (Madcity Labs, Nano LP-100). A scanned image of single-molecules (dye-labeled DNA) is shown in Fig. 1(b). With the optimal condition (focal spot inside water) our objective lens had a beam waist up to $\sim 200 \mathrm{~nm}$. As our sample is attached to a coverglass, the laser beam focusing is less than optimal, but should be less than $500 \mathrm{~nm}$. To control the surface density of dye-labeled DNA, we diluted the solution containing dye-labeled DNA enough to get the distance between the adjacent DNAs larger than several micrometers, so that we can put one molecule in the focal spot at a time. After finding the position of the molecules, each molecule was exposed for 30 seconds with $100 \mathrm{~ms}$ time resolution to obtain the time trace of fluorescence intensity of a FRET pair. All measurements were controlled by homebuilt software written in LabView.

\section{DNA and the fluorescent dyes}

Cy3 and Cy5 dyes [9] were used for the donor and acceptor, respectively, of which Forster radius is known to be $6 \mathrm{~nm}$ [10]. Merits of using Cy3-Cy5 FRET pairs arise from following facts; 1) spectral separation is large, 2) quantum yield is relatively high and comparable to each other, and 3) both are photo-stable in an oxygenfree environment. To give a nanometer-scale distance between the donor and acceptor dyes, we used a dye-labeled partial duplex DNA consisting of a poly(deoxythymidylic acid) $[\operatorname{poly}(\mathrm{dT})]$ attached to a double strand DNA of 18 base pairs (Fig. 2(a)). The partial duplex DNA was prepared by hybridization of two single strand DNA containing complementary sequences the sequence of

(a)

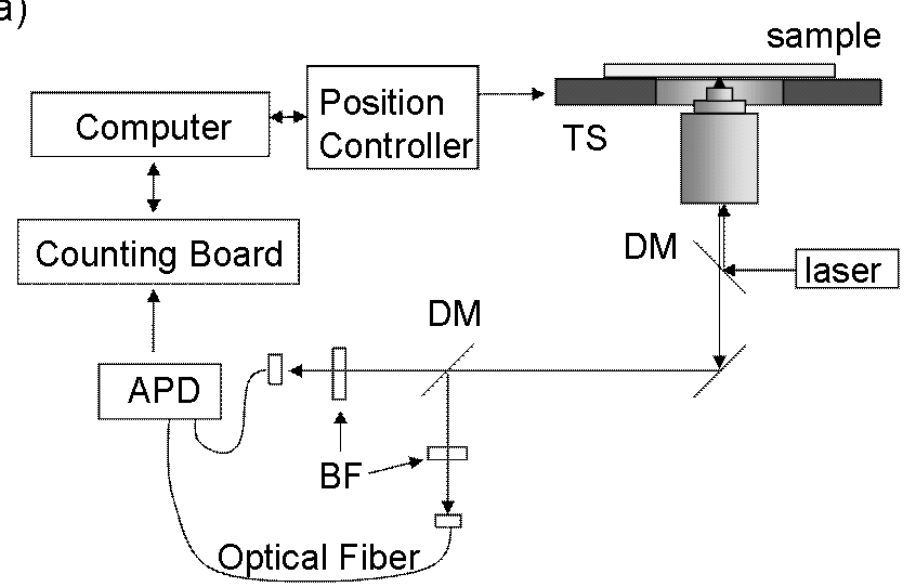

(b)

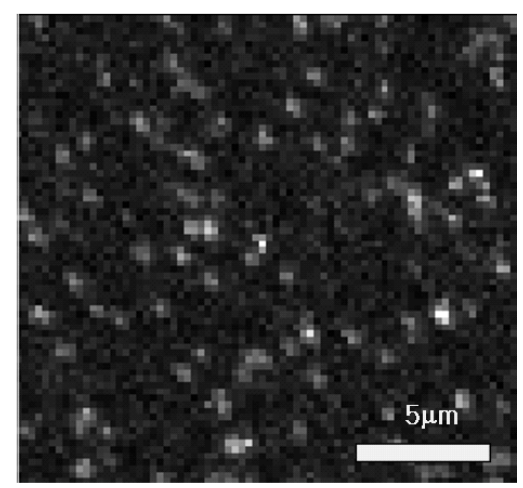

FIG. 1. (a) Schematic diagram of the setup based on confocal microscope. (APD: Avalanche photo diode, DM: Dichroic mirror, TS: Translation stage, and BF: Bandpass filter) (b) Scanned image of single molecules with a field size $20 \mu \mathrm{m}$ $\times 20 \mu \mathrm{m}$. Each bright spot in the image indicates the immobilized dye-labeled DNA. The size of the spots are about the diffraction limits $(<500 \mu \mathrm{m})$. 
(a)

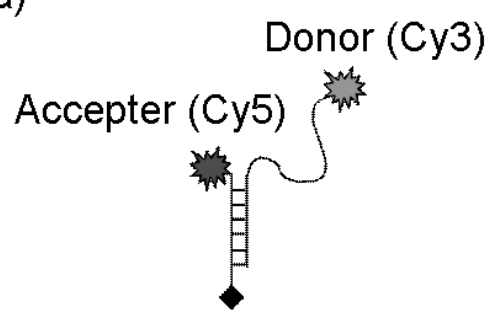

(b)

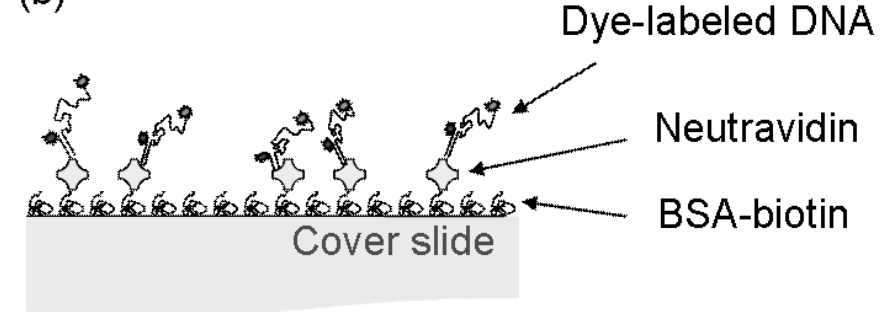

FIG. 2. (a) Structure of partial duplex DNA labeled with Cy3 and Cy5. (b) The partial duplex DNA attached to the cover slide via biotin-Nuetravidin-BSA linker.

cy3 labeled single strand DNA is 5'-TGG CGA CGG CAG CGA GGC-(dT) $)_{n}-\mathrm{Cy} 3$, and the sequence of cy5 labeled is 5'-Cy5-GCC TCG CTG CCG TCG CCA. Dye-labeled single strand DNA was purchased from IDTDNA and the dye labeling was made during DNA synthesis via phosphate backbone modification. As shown in Fig. 2(a), Cy3 was attached at the end of the 3 ' tail of the partial duplex DNA and an acceptor Cy5 was located at the junction of the double strand DNA and 3' single strand tail, so that the distance between the donor and acceptor could be manipulated by the number of the deoxythymidylic acids $(\mathrm{dT})_{\mathrm{n}} .10 \mathrm{mM}$ of Tris buffer with $\mathrm{pH} 8.0$ was used in the presence of 30 $\mathrm{mM}$ of $\mathrm{Na}^{+}$ion. All measurements were performed at room temperature.

\section{Sample chamber and immobilization of single- molecule}

The sample chamber with $100 \mu m$ thickness was prepared by sandwiching a cover slip and slide glass spaced by double-side tape. Two holes on the slide, drilled at the end of the sample chamber, were used for the inlet and outlet of samples. Quartz slides were sonicated in a glass staining dish filled with acetone for 20 minutes followed by 5 -minute sonication in water. Subsequent sonication in methanol and $1 \mathrm{M} \mathrm{KOH}$ was performed successively. DNA immobilization is realized through a neutravidin-biotin linker (Fig. 2(b)). $1 \mathrm{mg} / \mathrm{ml}$ of biotinylated albumin (Sigma) was first introduced to coat the glass surface. Then $0.25 \mathrm{mg} / \mathrm{ml}$ of neutravidin (Pierce) was injected into the channel followed by an incubation of $100 \mathrm{pM}$ of the biotinylated partial duplex DNA.

\section{Imaging buffer}

Dye photobleaching and the nonradiative triplet state are the main obstacles in the use of fluorescence dyes. For the Cy3-Cy5 FRET pair, it is known that the oxygen free environment in the presence of triplet quencher improves photo-stability of dyes greatly. To prevent the photobleaching of the dyes, an oxygen scavenging system consisting of $1 \mathrm{mg} / \mathrm{ml}$ glucose oxidase (Sigma), $0.8 \backslash \%$ (w/v) dextrose (Sigma), and $0.04 \mathrm{mg} / \mathrm{ml}$ catalase (Roche) was added into the buffer [11]. However, removal of oxygen significantly increases the photo-inactive triplet lifetime of dyes as the triplet oxygen acts as a triplet quencher of the dyes. To avoid this effect, $1 \%$ (v/v) 2-mercaptoethanol (Acros) was added to the buffer as they are known to be an effective triplet quencher [12].

\section{RESULT AND DISCUSSION}

A representative time trajectory of fluorescence intensities and FRET efficiency obtained from a single DNA molecule $(\mathrm{n}=12)$ is depicted in Fig. 3(a). Initially, the fluorescence from the acceptor was higher than that from the donor to yield a FRET value of $\sim 0.65$. The sudden drop of fluorescence intensity in the acceptor channel to its background level at 16 seconds is associated with an anti-correlated increase of the donor fluorescence indicating photobleaching of the acceptor dye. Similarly, photobleaching of the donor molecule was observed at 26 seconds. From these single photobleaching events, we could distinguish a single FRET pair from multi-labeled molecules that showed multiple photobleaching events as in Fig. 3(b). Since the overlap of the donor emission to acceptor emission causes measureable effect for accurate evaluation of the FRET efficiency, leakage correction must be considered in Eq. (2).

$$
E=\frac{F_{A}-F_{D} \times L}{F_{A}+F_{D}}
$$

where $L$ is a leakage correction factor for the contribution of the donor fluorescence to the acceptor channel. Leakage of the donor signal to the acceptor channel can be determined by calculating the ratio of the acceptor channel to the donor channel after acceptor photobleaching. More precise calculation can be carried out by considering the background signals as follows.

$$
E=\frac{F_{A}-B_{A}-\left(F_{D}-B_{D}\right) \times L}{F_{A}-B_{A}+F_{D}-B_{D}}
$$


(a)

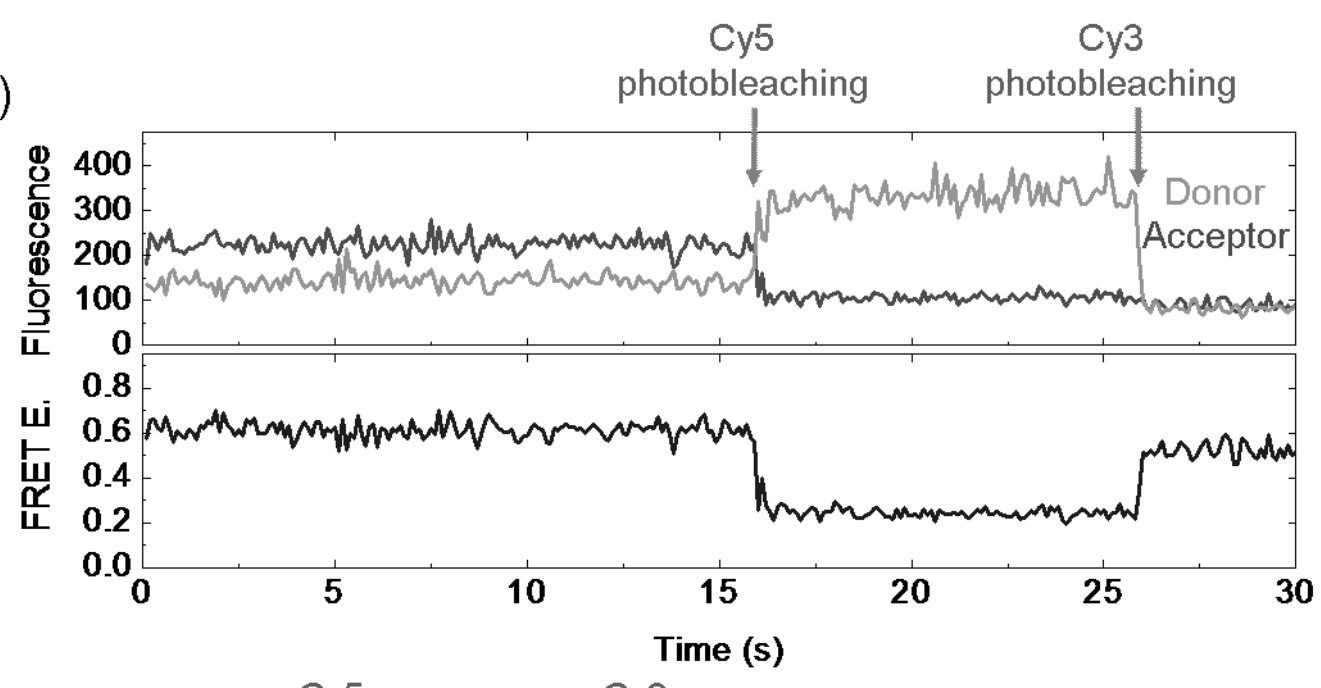

(b)

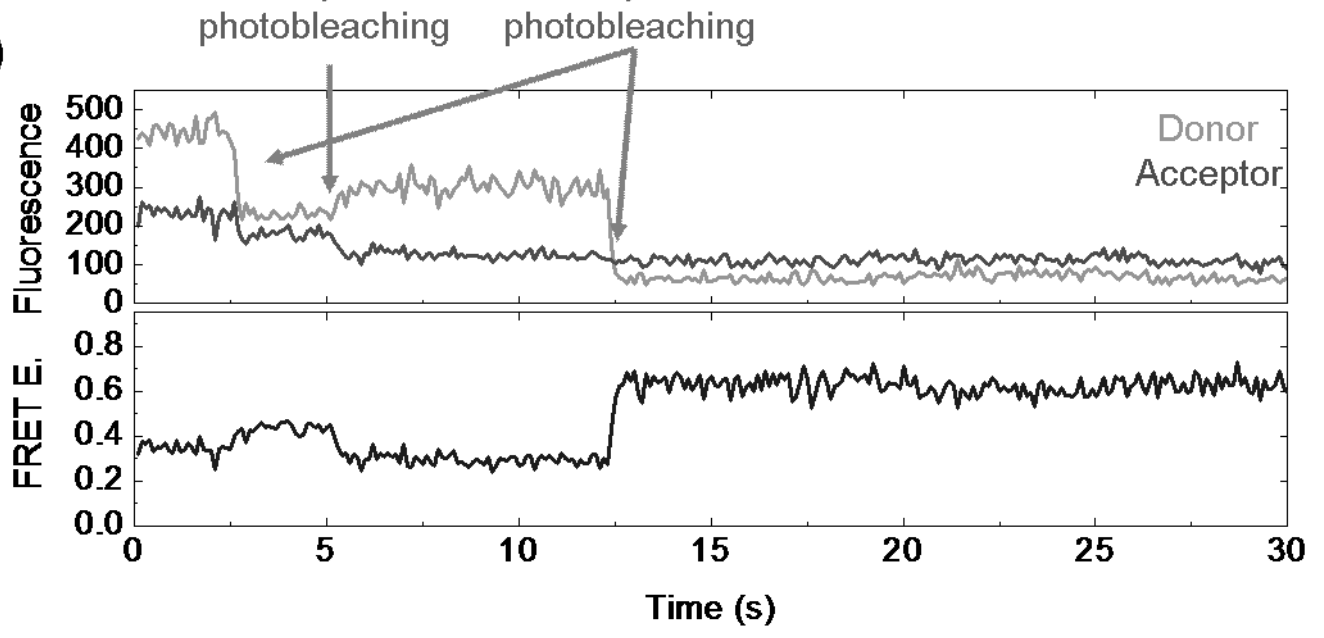

FIG. 3. (a) A representative single-molecule time trajectory of fluorescence intensities and FRET efficiency. (b) Time trace from two FRET pairs showing multiple photobleaching events.

where $\mathrm{B}_{A}$ and $\mathrm{B}_{D}$ are background counts for each channel. The background could be determined by the photon counts measured after dye photobleaching.

We then compared the FRET efficiency for different inter-dye distances adjusted by the length of single strand overhang. We built FRET histograms to get a statistical information of the FRET pairs, as shown in Figs. 4(a) and (b) for $(\mathrm{dT})_{12}$ and $(\mathrm{dT})_{30}$, respectively. For the FRET histograms, first ten data points of each FRET time trajectory were averaged for each molecule and used as a representative FRET value of the molecule. Since the conformational change of the single strand DNA and corresponding change of the orientation between two dye molecules is known to be much faster than our time resolution of $\sim 100 \mathrm{~ms}$ [10], average over ten data points (corresponding to 1 second) is a good approximation to assign the FRET value of the molecules at a certain moment. However, if the system has a dynamics of which the time scale is comparable to the time resolution, one can build a histogram with all the data points to achieve good statistics. Shown in Figs. 4(c) and (d) are leakage and background corrected FRET histograms of single FRET pairs of $(\mathrm{dT})_{12}$ and $(\mathrm{dT})_{30}$, respectively. Peaks at around 0.67 for $(\mathrm{dT})_{12}$ and 0.23 for $(\mathrm{dT})_{30}$ were found in the histograms, indicating the donor and acceptor dyes on $(\mathrm{dT})_{30}$ are further apart. The peak found at 0.5 for $(\mathrm{dT})_{12}$ histogram is due to the donor- and acceptor-bleached molecules, and the peak at 0.0 for $(\mathrm{dT})_{30}$ is acceptor-bleached or donor-only labeled molecules. The distances between the donor and the acceptor calculated from the measured FRET value were $5.33 \mathrm{~nm}$ and $7.33 \mathrm{~nm}$ for $(\mathrm{dT})_{12}$ and $(\mathrm{dT})_{30}$, respectively. 

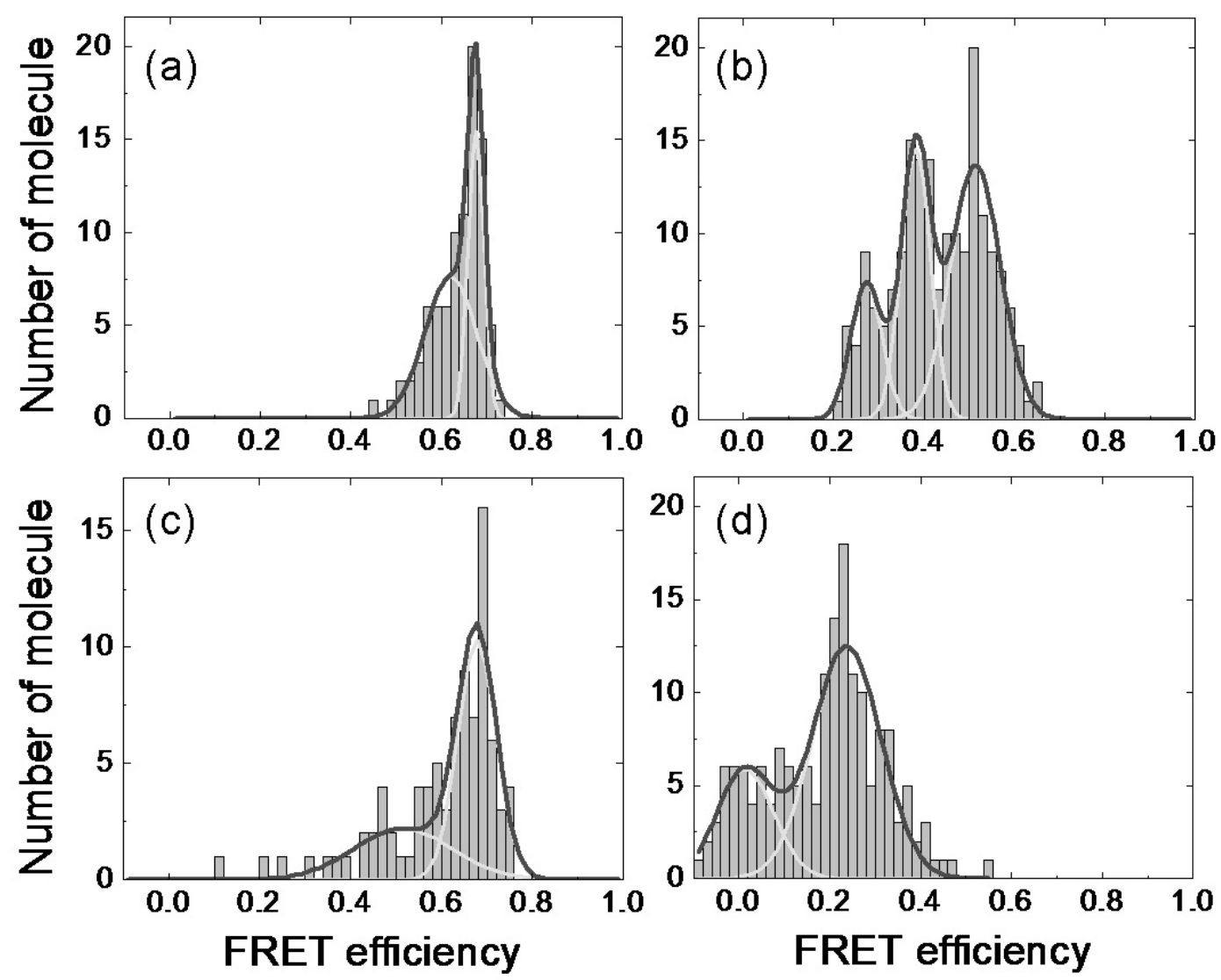

FIG. 4. FRET histograms of molecules having (a) (dT) $)_{12}$, and (b) (dT) $)_{30}$. Leakage and background corrected histograms for $(\mathrm{c})(\mathrm{dT})_{12}$ and $(\mathrm{d})(\mathrm{dT})_{30}$.

\section{CONCLUSIONS}

In summary, we have successfully measured FRET from single donor-acceptor pairs using confocal microscopy. Single photobleaching events shown in the FRET time trajectory assured the detection of the single-molecule. As the single-molecule FRET technique provides real time observation of inter- and intra molecular dynamics of macromolecules in nanometer scale, we expect that the technique can be used for biological applications such as identifying short-lived states during enzyme activity. The technique is also very promising fordetection or characterization of the small quantity of targets that are required for this small device [13].

\section{ACKNOWLEDGEMENT}

This work was supported by Seoul Research and Business Development Program (10816) and the Quantum Photonic Science Research Center (SRC) at Hanyang University.

\section{REFERENCES}

[1] T. Forster, Ann. Phys., 2, 55, 1948.

[2] P. V. Cornish, and T. Ha, ACS Chem. Biol. 2, 53, 2007.

[3] T. Ha, Biochemistry 43, 4055, 2004.

[4] X. Zhuang, Annu. Rev. Biophys. Biomole. Strut. 34, 399, 2005.

[5] G. W. Gordon, G. Berry, X. H. Liang, B. Levine, and B. Herman, Biophys. J. 74, 2702, 1998.

[6] T. Mori, R. D. Vale, and M. Tomishige, Nature 450, $750,2007$.

[7] T. Ha, A. Y. Ting, J. Liang, W. B. Caldwell, A. A. Deniz, D. S. Chemla, P. G. Schultz, and S. Weiss, Proc. Natl. Acad. Sci. 96, 893, 1999.

[8] T. Ha, D.S. Chemla, Th. Enderle, and S. Weiss, Appl. Phys. Lett. 70, 782, 1997.

[9] P. L. Southwick, L. A. Ernst, E. W. Tauriello, S. R. Parker, R. B. Mujumdar, S. R. Mujumdar, H. A. Clever, and A. S. Waggoner, Cytometry 11, 418, 1990.

[10] M. C. Murphy, I. Rasnik, W. Cheng, T. M. Lohman, and T. Ha, Biophys. J. 86, 2530, 2004.

[11] R. E. Benesch, and R. Benesch, Science 118, 447, 1953.

[12] I. Rasnik, S. McKinney, and T. Ha, Nat. Meth. 34, 891, 2006. 